\title{
Uso de la Gamificación como metodología activa en la Educación secundaria española durante la pandemia por COVID-19
}

\author{
Julián Roa González - Universidad a Distancia de Madrid (UDIMA) \\ Almudena Sánchez Sánchez - Universidad a Distancia de Madrid (UDIMA) \\ Natalia Sánchez Sánchez - Universidad a Distancia de Madrid (UDIMA) \\ Verónica Basilotta Gómez-Pablos - Universidad a Distancia de Madrid (UDIMA)

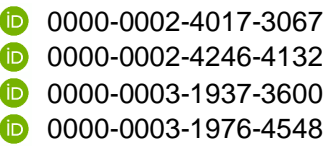

Recepción: 21.01.2022 | Aceptado: 31.01.2022

Correspondencia a través de ORCID: Almudena Sánchez Sánchez

iD 0000-0002-4246-4132

Citar: Roa González, J, Sánchez Sánchez, A, Sánchez Sánchez, N y Basilotta Gómez-Pablos, V (2022). Uso de la Gamificación como metodología activa en la Educación secundaria española durante la pandemia por COVID-19. REIDOCREA, 11(10), 105-119.

Estudio de investigación del Observatorio de la Innovación Educativa de la UDIMA.

Área o categoría del conocimiento: Multidisciplinar

Resumen: El empleo de metodologías activas es un elemento clave en la innovación educativa que ha sido evaluado por el Observatorio de Innovación Educativa de la Universidad a Distancia de Madrid (Universidad Abierta) desde su creación. Entre estas metodologías activas destaca la gamificación, tanto por su potencial como por su interés mediático. La investigación en educación ha aportado claridad en cuanto a sus características y elementos esenciales, destacando sus posibilidades y ventajas como elemento dinamizador de los procesos de enseñanza-aprendizaje. El objetivo de este artículo es ofrecer un análisis cuantitativo del porcentaje de uso de la gamificación en el primer semestre del curso 2020-2021 en la educación secundaria española, e identificar las variables que influyen en su uso e implementación. Para ello, se ha empleado un muestreo no estratificado a través de cuestionarios que ha permitido analizar el uso de la gamificación en 177 aulas de secundaria y bachillerato. En general, los datos recogidos y analizados en este estudio sugieren que la gamificación es una metodología en auge tras la situación excepcional de confinamiento, aunque se utiliza como metodología de apoyo y su implantación en las instituciones de educación secundaria españolas no está suficientemente consolidada. Además, variables tipo como escuela, especialidad, años de experiencia docente y formación pedagógica constituyen valores significativos a la hora de implementar la metodología.

Palabra clave: Gamificación

Use of Gamification as an active methodology in Spanish Secondary Education during the COVID19 pandemic

Abstract: Employing active methodologies is a key element in educational innovation that has been
assessed by the Observatory of Educational Innovation of the Universidad a Distancia de Madrid (Open
University) since its establishment. Gamification stands out among these active methodologies, both in terms
of its potential as well as because of its media interest. Research in education has brought about clarity
regarding its characteristics and essential elements, highlighting its possibilities and advantages as a
dynamizing element in teaching-learning processes. The goal of this article is to provide a quantitative
analysis of the percentage of use of gamification in the first semester of the $2020-2021$ course in Spanish
secondary education, and to identify variables that influence its use and implementation. To this end, non-
stratified sampling through questionnaires has been employed to enable the analysis of the use of
gamification in 177 secondary and upper-secondary classrooms. In general, the data gathered and analyzed
in this study suggests that gamification is a methodology on the rise after the exceptional situation of the
lockdown, although it is used as a supporting methodology and its implementation in Spanish secondary
education institutions is insufficiently established. Furthermore, type variables such as school, specialty,
years of teaching experience and pedagogical training constitute significant values when implementing the
methodology.

Keyword: Gamification 


\section{Introducción}

La situación derivada de la pandemia por COVID 19 ha llevado a los diferentes países a modificar parcialmente la organización de sus sistemas educativos. En España, el primer semestre del curso 2020-21 se ha llevado a cabo bajo una docencia presencial, con períodos excepcionales de semipresencialidad, que ha implicado una reducción de las ratios educativas y una reestructuración de los centros para cumplir con las normas básicas sanitarias.

Estos planteamientos organizativos derivados de la pandemia deben conjugarse con la legislación educativa española. Según se indica en el Preámbulo de la Ley Orgánica 8/2013, de 9 de diciembre, para la mejora de la calidad educativa, "EI sistema educativo debe posibilitar tanto el aprendizaje de cosas distintas como la enseñanza de manera diferente, para poder satisfacer a unos alumnos y alumnas, que han ido cambiando con la sociedad. Las habilidades cognitivas, siendo imprescindibles, no son suficientes; es necesario adquirir desde edades tempranas competencias transversales". Las escuelas deben, por tanto, cumplir con una doble responsabilidad: por un lado, deben ofrecer un entorno seguro de trabajo y por otro deben seguir apostando por la innovación educativa que permita el desarrollo de los contenidos y las competencias claves previstas en el currículo.

Dentro de este contexto, la innovación en metodologías activas que se venía realizando ha sufrido cambios respecto a los cursos precedentes. Algunas metodologías como el aprendizaje cooperativo son más difíciles de implementar en las aulas al requerir el contacto estrecho entre estudiantes. Otras metodologías sustentadas por las TIC son más proclives a una aplicación que respete las normas sanitarias. En este trabajo nos centraremos en la metodología activa de la gamificación encontrando referencias a esta nueva metodología desde hace 10 años (Deterding et al 2011; Kapp, 2012; Marczewski, 2013; Werbach y Hunter, 2012; Zichermann y Cunningham, 2011). Apoyándonos en estos trabajos podemos definir la gamificación como un método en el que se utilizan mecánicas del juego en contextos de la vida cotidiana para influir en las personas que las utilizan.

Es importante señalar la diferencia entre gamificación, aprendizaje basado en juegos y serious games. Aunque los tres conceptos comparten el pensamiento lúdico como elemento esencial, en Marczewski (2015) y Ordás (2018) se recogen diferencias importantes. Así, los serious games se basan en la creación de videojuegos específicos con un fin no lúdico. El aprendizaje basado en juegos utiliza juegos existentes para contribuir a la enseñanza de los conceptos y competencias curriculares. En cambio, la gamificación incorpora algunos elementos del juego para aprender.

La investigación educativa ha establecido los elementos que utilizan los juegos, diferenciando entre dinámicas, mecánicas y componentes (Alejaldre y García, 2015; Werbach y Hunter, 2015; Wiklund y Wakerius, 2016). Siguiendo a estos autores, podemos acotar estos tres conceptos del juego. En un primer plano aparecen las dinámicas, elementos no tangibles pero que influyen en la motivación de los participantes y que parten de las necesidades e inquietudes 
humanas. En un segundo plano se encuentran los componentes que regulan el juego y establecen su funcionamiento y que sirven fundamentalmente para impulsar la acción; este segundo plano se denomina mecánicas. Por último, aparecen los elementos más visibles y tangibles como los puntos, las insignias, los combates o las clasificaciones que sirven para establecer una dinámica positiva cumpliendo con la mecánica del juego y que se denominan componentes.

Como todas las metodologías, la gamificación presenta ventajas e inconvenientes que han sido estudiadas por diferentes autores. Según Zichermann y Cunningham (2011), Kapp (2012) y Bruder (2015), las principales ventajas están relacionadas con el incremento motivacional, el uso de un ambiente de aprendizaje que favorece la búsqueda de nuevas soluciones y la resolución de retos, el aumento de la cooperación, la responsabilidad sobre el propio aprendizaje y la mejora en la retención de los conocimientos. En el otro extremo, y siguiendo el trabajo de Pérez de Villaamil (2018), las principales desventajas son el elevado coste en tiempo y recursos para crear el material, las distracciones asociadas al juego, la desmotivación una vez superada la novedad inicial y las dificultades en la adquisición de determinadas habilidades básicas como la expresión oral.

Tras una primera fase inicial de delimitación teórica en los últimos cinco años, numerosos estudios (Amezcua y Amezcua, 2018; Díaz, 2017; Gallego y Ágredo, 2016; González, Olivares, García, 2016; García y Figueroa, 2017; Ortiz, 2017; Pérez et al, 2017; Quintanal, 2016) han dado cuenta de experiencias gamificadas de aula en España. Adicionalmente, en Roa et al (2021) podemos encontrar una primera evaluación del grado de implantación de la gamificación en la Enseñanza Secundaria y el Bachillerato antes de la Pandemia por COVID-19.

\section{Objetivos}

El presente estudio pretende evaluar la situación de la implantación de la gamificación en las aulas de secundaria y bachillerato en el contexto de un curso educativo que se ha impartido durante la pandemia por COVID 19. Para ello, se analizan los datos recogidos en una encuesta realizada a estudiantes del máster de formación del profesorado tras finalizar su periodo de prácticas, que ha permitido analizar la implantación observada de esta metodología en 177 aulas de secundaria y bachillerato con currículo español durante el primer semestre del curso 2020-2021.

La investigación se ha centrado en conocer la implementación de la gamificación en la educación secundaria española dentro del contexto excepcional vivido durante la pandemia por COVID-19. De forma concreta se busca dar respuesta a los siguientes objetivos:

- Analizar y proporcionar datos cuantitativos acerca del uso de la gamificación como metodología en la educación secundaria española durante el primer semestre del curso 2020-21.

- Detectar variables que influyan significativamente en la implantación y utilización de la gamificación como metodología innovadora. 


\section{Método}

\section{Participantes}

El estudio se llevó a cabo entre octubre de 2020 y enero de 2021. Se obtuvieron 177 respuestas de 89 estudiantes del Máster Universitario en Formación del profesorado de Educación Secundaria que habían terminado sus prácticas presenciales en centros educativos de educación secundaria con currículo español. Los estudiantes tenían la oportunidad de responder a 3, 2 o 1 cuestionarios en función de si habían realizado sus prácticas en clases de primer ciclo de la ESO, en clases de segundo ciclo de la ESO o en clases de Bachillerato, esto explica por qué el número de aulas observadas es superior al número de estudiantes. Los profesores en formación encuestados se distribuían de forma equilibrada por sexos (52,80\% mujeres, $48,20 \%$ hombres) de los cuales $4,49 \%$ son menores de 25 años, $77,53 \%$ tienen una edad comprendida entre 25 y 40 años (ambos inclusive) y el 17,98\% restante son mayores de 40 años. Inicialmente, el cuestionario fue respondido por 101 estudiantes, pero se excluyeron las respuestas de doce estudiantes que indicaron que no deseaban que sus respuestas se incluyeran en este estudio.

Los participantes del estudio pertenecen a cuatro especialidades diferentes dentro de los estudios de Máster: Matemáticas (42.69\%), Lengua y literatura (7.86\%), Lengua extranjera (24.73\%) y Geografía e Historia (24.72\%). En cuanto al tipo de centro, los participantes en el estudio pertenecen a tres tipos de centro diferentes: colegio privado $(14,7 \%)$, concertado $(53,0 \%)$ y público $(32,3 \%)$. Adicionalmente, se han recogido datos generales sobre los/as tutores/as que supervisaban las prácticas en los centros educativos. El $24,72 \%$ de los/as tutores/as tenían una experiencia docente de entre 1 y 5 años (ambos inclusive), el 20,22\% una experiencia de entre 6 y 10 años (ambos inclusive) y el resto una experiencia mayor de 10 años (55,06\%); con edades comprendidas entre 25 y 40 años (ambos inclusive) un 34,83\% y el resto mayores de 40 años $(65,17 \%)$. Así mismo, respecto a la formación pedagógica de los/as tutores, un $66,29 \%$ realizaron el CAP mientras el 25,84\% realizaron el Máster de Formación del Profesorado; el resto o no tienen formación pedagógica o han realizado otra formación diferente (5,62\% y 2,25\%, respectivamente). Asociado a los cursos en los que fueron realizadas las prácticas, teniendo en cuenta que un estudiante puede realizar sus prácticas en los distintos ciclos educativos, el $38,98 \%$ de las sesiones de prácticas fueron realizadas en el primer ciclo académico $\left(1^{\circ}, 2^{\circ} \circ 3^{\circ}\right.$ ESO); el 30,51\% realizaron sesiones en el $2^{\circ}$ Ciclo (4ํㅡㄴ $\mathrm{ESO}$ ); el $24,86 \%$ las realizaron en Bachillerato y el resto en centros de FP (5,65\%). Por último, asociado a los tipos de espacios empleados para el desarrollo de las clases, resaltar que un elevado porcentaje de las sesiones fueron realizadas en una tipología de aula tipo aulario (84,18\%) mientras que se empleó Aula Abierta, Aula de estructura variables y aula materia el $2,26 \%, 5,65 \%$ y $5,65 \%$ de las ocasiones, respectivamente. El resto $(2,26 \%)$ indicaron que se empleó otro tipo de aula no definido. 


\section{Instrumentos}

Los datos se recopilaron mediante un cuestionario informatizado del que se obtenían datos estadísticos generales e información específica sobre la innovación educativa observada.

El cuestionario ha sido elaborado por los profesores del Observatorio de Innovación Educativa de la Universidad a Distancia de Madrid y ha sido aprobado para su uso por el Comité de Ética de dicha Universidad. Cuenta con 30 items que pueden cumplimentarse en menos de 30 minutos.

\section{Procedimiento}

Una vez recogidos los datos, durante los meses de febrero y marzo de 2021 se han seguido las siguientes fases para codificar los datos:

1. Unificación de las respuestas aportadas. La variable objeto de estudio presentaba 5 posibilidades de implantación graduada en función del porcentaje de aplicabilidad de la metodología y que, a efectos del análisis estadístico, ha sido dicotomizada con las siguientes opciones:

- Centro que no emplea la gamificación como metodología o lo hace de forma superficial.

- Centro que emplea la gamificación como metodología en un porcentaje de las sesiones superior al $25 \%$.

2. Análisis cuantitativo de los datos. Aplicando técnicas básicas de estadística descriptiva, en particular, tablas de contingencia, se han obtenido los diferentes grados de implantación de la metodología teniendo en cuenta las opciones de respuesta proporcionadas en la fase anterior.

3. Análisis comparativo de medias no paramétrico. Aplicando, en su caso, test de Kruskal Wallis (Kruskal y Wallis, 1952) y estadístico de Mann-Whitney (McKnight y Najab, 2010) para determinar la posible existencia de diferencias significativas entre grupos y, en particular, dónde se encuentran dichas diferencias.

Los análisis estadísticos fueron realizados con el software estadístico $R$ Version 1.0.136. En primer lugar, se halló una estadística descriptiva del ítem asociado a la metodología activa analizada. En segundo lugar, se testó la normalidad de la distribución comprobándose que no se cumplía (prueba de KolmogorovSmirnov <.05) (Berger y Zhou, 2014). Debido a la falta de normalidad, en los análisis siguientes se emplearon pruebas no paramétricas: prueba de Kruskal Wallis para medidas independientes, tomando el ítem asociado a la metodología empleada como variable dependiente y considerando las variables estudiadas (ciclo educativo, formación pedagógica, edad y años de experiencia del tutor, tipo de centro, tipo de espacio empleado y especialidad cursada) como variables de agrupación. 


\section{Resultados}

La investigación realizada durante el primer semestre del curso 2020-21 ha permitido conocer el grado de implantación de la gamificación en los centros de Educación secundaria españoles durante el primer semestre de clases con restricciones por la pandemia de COVID-19. Presentamos a continuación las siguientes tablas de resultados.

En la Tabla 1, se muestran los estadísticos descriptivos (media, varianza y porcentajes) del ítem asociado al uso de la gamificación en las aulas analizadas. Los resultados muestran que el $80.23 \%$ de los estudiantes observan que la gamificación no forma parte de las metodologías empleadas o se emplea de modo muy superficial en su centro mientras que el $19.77 \%$ indica que tiene una aplicabilidad de más del $25 \%$ de las sesiones observadas.

Tabla 1. Estadísticos descriptivos de la variable "uso de la Gamificación (GA)".

\begin{tabular}{lccccc}
\hline & Media & Desviación típica & Nada o de forma superficial & $>25 \%$ de las sesiones & Total \\
\cline { 2 - 6 } $\begin{array}{l}\text { He visto que la GA en el aula } \\
\text { observadas emplea }\end{array}$ & 0,2 & 0,4 & $80,23 \%$ & $19,77 \%$ & $100 \%$ \\
\hline
\end{tabular}

En la Tabla 2 se muestran los estadísticos descriptivos (media, desviación típica y porcentajes) asociados al uso de la Gamificación según las especialidades de los profesores en formación. Como se puede apreciar en la figura 1, destaca el escaso uso de la metodología en la especialidad de Matemáticas $(2,86 \%)$ y el uso moderado que se realiza en el resto de las especialidades de Geografía e Historia (25,53\%), Lengua extranjera inglés $(36,36 \%)$ y Lengua y Literatura $(31,25 \%)$.

Tabla 2. Estadísticos descriptivos de la variable "uso de la Gamificación (GA) en las distintas especialidades".

\begin{tabular}{lccccc}
\hline & Media & $\begin{array}{c}\text { Desviación } \\
\text { típica }\end{array}$ & $\begin{array}{c}\text { Nada o de forma } \\
\text { superficial (\%) }\end{array}$ & $\begin{array}{c}>25 \% \text { de las } \\
\text { sesiones (\%) }\end{array}$ & $\begin{array}{c}\text { Total } \\
(\%)\end{array}$ \\
\cline { 2 - 6 } $\begin{array}{l}\text { He visto que la GA en el aula de } \\
\text { Geografía e Historia se emplea }\end{array}$ & 0,26 & 0,44 & 74.47 & 25.53 & 100 \\
\hline $\begin{array}{l}\text { He visto que la GA en el aula de } \\
\text { Lengua extranjera inglés se emplea }\end{array}$ & 0,36 & 0,49 & 63.64 & 36.36 & 100 \\
\hline $\begin{array}{l}\text { He visto que la GA en el aula de } \\
\text { Lengua y Literatura se emplea }\end{array}$ & 0,31 & 0,48 & 68.75 & 31.25 & 100 \\
\hline $\begin{array}{l}\text { He visto que la GA en el aula de } \\
\text { Matemáticas se emplea }\end{array}$ & 0,03 & 0,17 & 97.14 & 2.86 & 100 \\
\hline
\end{tabular}




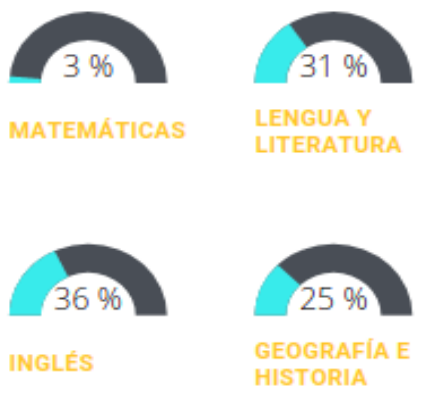

Figura 1. Uso de la Gamificación en función de las especialidades.

A continuación, se ha realizado un desglose de los datos generales en función del tipo de centro educativo (Tabla 3) y perfil del tutor. En la Figura 2 se muestran los resultados obtenidos para la enseñanza privada, concertada y pública. Resalta que el uso de la gamificación no es una metodología dominante en ninguna de las tipologías de centro. Sin embargo, parece que en la enseñanza privada y concertada hay una tendencia a utilizar esta metodología en torno al $27.17 \%$ y $20 \%$ de las aulas observadas, respectivamente, mientras que en la enseñanza pública sigue siendo una metodología muy poco empleada (9.23\%) en las aulas analizadas.

Tabla 3. Estadísticos descriptivos de la variable "uso de la Gamificación (GA) en los distintos tipos de centros".

\begin{tabular}{lccccc}
\hline & Media & Desviación típica & $\begin{array}{c}\text { Nada o de forma } \\
\text { superficial (\%) }\end{array}$ & $\begin{array}{c}>25 \% \text { de las } \\
\text { sesiones (\%) }\end{array}$ & Total (\%) \\
\cline { 2 - 5 } $\begin{array}{l}\text { He visto que la GA en el aula de } \\
\text { colegios concertados se emplea }\end{array}$ & 0,27 & 0,45 & 72.83 & 27.17 & 100 \\
\hline $\begin{array}{l}\text { He visto que la GA en el aula de } \\
\text { colegios privados se emplea }\end{array}$ & 0,2 & 0,41 & 80 & 20 & 100 \\
\hline $\begin{array}{l}\text { He visto que la GA en el aula de } \\
\text { colegios públicos se emplea }\end{array}$ & 0,09 & 0,29 & 90,77 & 9,23 & 100 \\
\hline
\end{tabular}

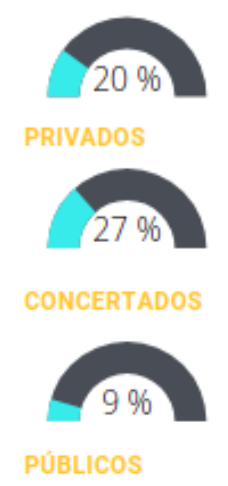

Figura 2. Uso de la Gamificación en función de la tipología de centro.

En Tabla 4 se muestran los estadísticos descriptivos del uso de la gamificación, esta vez, correspondientes a cada ciclo educativo (1ํ. $2^{\circ}$, $3^{\circ}$ ․ ESO; $4^{\circ}$ ESO; Bachillerato). De acuerdo a los resultados obtenidos (Figura 3), destacar el bajo uso de la metodología en Bachillerato (15,91\%) siendo ligeramente superior en el resto de los niveles educativos (primer ciclo ESO, 21,74\%; 4을. 
Tabla 4. Estadísticos descriptivos de la variable "uso de la Gamificación (GA) en los distintos niveles educativos".

\begin{tabular}{lccccc}
\hline & Media & $\begin{array}{c}\text { Desviación } \\
\text { típica }\end{array}$ & $\begin{array}{c}\text { Nada o de forma } \\
\text { superficial }\end{array}$ & $>25 \%$ de las sesiones & Total \\
\cline { 2 - 5 } $\begin{array}{l}\text { He visto que la GA en el aula de } \\
\text { primer ciclo de la ESO se emplea }\end{array}$ & 0,22 & 0,42 & 78,26 & 21,74 & 100 \\
\hline $\begin{array}{l}\text { He visto que la GA en el aula de } \\
\text { segundo ciclo de la ESO se emplea }\end{array}$ & 0,2 & 0,41 & 79,63 & 20,37 & 100 \\
\hline $\begin{array}{l}\text { He visto que la GA en el aula de } \\
\text { Bachillerato se emplea }\end{array}$ & 0,16 & 0,37 & 84,09 & 20 & 100 \\
\hline
\end{tabular}

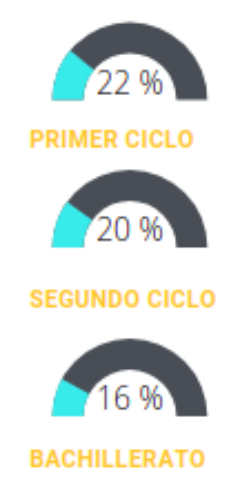

Figura 3. Uso de la Gamificación en función del ciclo educativo. Elaboración propia

En el siguiente bloque, se ha realizado un análisis acerca del perfil del tutor/a del centro de prácticas, en particular, los años de experiencia, la formación pedagógica y la edad del tutor/a con el fin de determinar, si existen diferencias en el uso de la gamificación en función de estas variables. En la Tabla 5 se muestran los resultados obtenidos en función de los años de experiencia docente. Destaca que los/las tutores/as con experiencia docente de entre 6 y 10 años presentan un mayor porcentaje en la aplicación de la metodología, en torno al $32,35 \%$, mientras que los que tienen menos de 5 años y más de 10 presentan unos porcentajes de uso de la metodología inferiores; 19,51 y 15,69\%, respectivamente.

Tabla 5. Estadísticos descriptivos de la variable "uso de la Gamificación (GA) de acuerdo con los años de experiencia del tutor".

\begin{tabular}{lccccc}
\hline & Media & Desviación típica & Nada o de forma superficial & $>25 \%$ de las sesiones & Total \\
\cline { 2 - 6 } $\begin{array}{l}\text { Entre 1 y 5 años } \\
\text { (ambos inclusive) }\end{array}$ & 0,2 & 0,4 & $80,49 \%$ & $19,51 \%$ & $100 \%$ \\
\hline $\begin{array}{l}\text { Entre 6 y 10 años } \\
\text { (ambos inclusive) }\end{array}$ & 0,32 & 0,47 & $67,65 \%$ & $32,35 \%$ & $100 \%$ \\
\hline Más de 10 años & 0,16 & 0,37 & $84,31 \%$ & $15,69 \%$ & $100 \%$ \\
\hline
\end{tabular}

Con respecto a la formación pedagógica del tutor/a, tal y como se muestra en Tabla 6, En función a los datos recogidos destaca que la gamificación es usada en torno al $29,17 \%$ por aquellos/as tutores/as que han cursado el máster del profesorado mientras que aquellos/as tutores/as que se formaron mediante el CAP tan solo lo emplean un $14,16 \%$. 
Tabla 6. Estadísticos descriptivos de la variable "uso de la Gamificación (GA) de acuerdo con la formación pedagógica realizada por el tutor de prácticas".

\begin{tabular}{lccccc}
\hline & Media & Desviación típica & Nada o de forma superficial & $>25 \%$ de las sesiones & Total \\
\cline { 2 - 6 } CAP & 0,14 & 0,35 & $85,84 \%$ & $14,16 \%$ & $100 \%$ \\
\hline $\begin{array}{l}\text { Máster de Formación } \\
\text { del Profesorado }\end{array}$ & 0,29 & 0,46 & $70,83 \%$ & $29,17 \%$ & $100 \%$ \\
\hline
\end{tabular}

El uso de la metodología de Gamificación respecto a la edad del/de la tutor/a muestra que los/las tutores/as de entre 25 y 40 años emplean en torno al 34,38\% la metodología frente a un $11,5 \%$ de uso en aquellos que tienen más de 40 años (véase Tabla 7).

Tabla 7. Estadísticos descriptivos de la variable "uso de la Gamificación (GA) de acuerdo con la edad del tutor de prácticas".

\begin{tabular}{lccccc}
\hline & Media & $\begin{array}{c}\text { Desviación } \\
\text { típica }\end{array}$ & $\begin{array}{c}\text { Nada o de forma } \\
\text { superficial (\%) }\end{array}$ & $\begin{array}{c}\mathbf{2 5 \%} \text { de las } \\
\text { sesiones (\%) }\end{array}$ & $\begin{array}{c}\text { Total } \\
(\%)\end{array}$ \\
\hline $\begin{array}{l}\text { Entre 25 y } 40 \text { años } \\
\text { (Ambos inclusive) }\end{array}$ & 0,34 & 0,48 & 65,62 & 34,38 & 100 \\
\hline Más de 40 años & 0,12 & 0,32 & 88,5 & 11,5 & 100 \\
\hline
\end{tabular}

En la Figura 4. Podemos ver de forma gráfica las variables estudiadas en relación con el profesor responsable del aula.

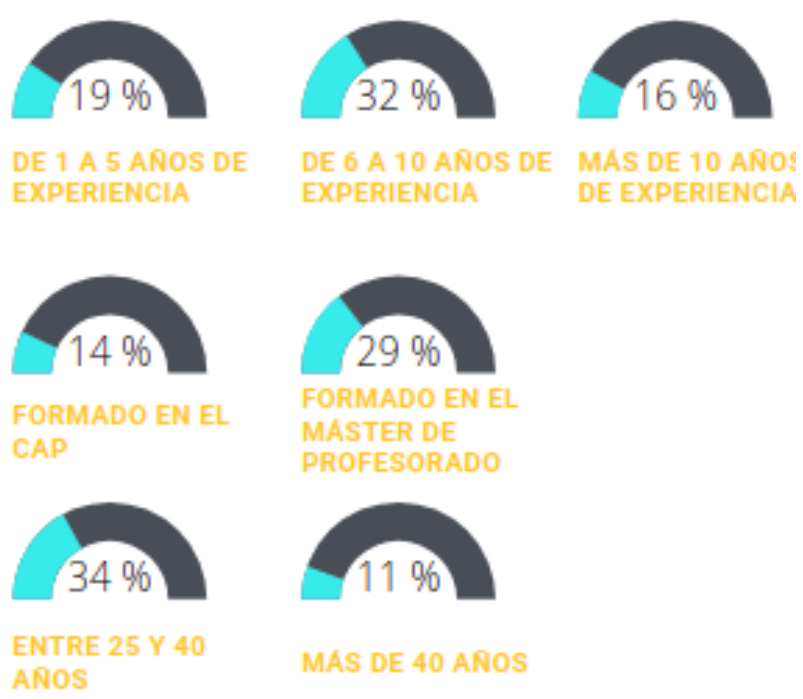

Figura 4. Uso de la Gamificación en función del profesor responsable del aula.

Para finalizar el análisis descriptivo, en la Tabla 8 se presentan los resultados obtenidos acerca del uso de la Gamificación en el aula en relación con los tipos de espacios de aprendizaje empleados. En este caso se observa un menor uso de la metodología en los espacios de aprendizaje tipo aulario siendo más empleado, sin embargo, en el resto de las tipologías de aula. No obstante, es importante señalar que el predominio en el uso de aularios en la muestra estudiada $(84,18 \%)$ hace que los datos relativos al resto de espacios de aprendizaje se basen en una muestra muy pequeña. 
Tabla 8. Estadísticos descriptivos de la variable "uso de la Gamificación (GA) de acuerdo al espacio de aprendizaje empleado".

\begin{tabular}{lccccc}
\hline & Media & Desviación típica & $\begin{array}{c}\text { Nada o de forma } \\
\text { superficial }(\%)\end{array}$ & $\begin{array}{c}>25 \% \text { de las } \\
\text { sesiones (\%) }\end{array}$ & Total (\%) \\
\cline { 2 - 5 } Aula abierta & 0,25 & 0,5 & 75 & 25 & 100 \\
\hline $\begin{array}{l}\text { Aula de estructura variable por } \\
\text { agrupamiento }\end{array}$ & 0,2 & 0,42 & 80 & 20 & 100 \\
\hline Aula Materia & 0,3 & 0,48 & 70 & 30 & 100 \\
\hline Aulario & 0,19 & 0,39 & 81,21 & 18,79 & 100 \\
\hline
\end{tabular}

A continuación, nos interesa analizar si existen diferencias significativas en el uso de la metodología de Gamificación en función de las variables expuestas previamente.

Tras la aplicación de la prueba Kruskal Wallis, con un nivel de significación de $\alpha=0,05$ no encontramos diferencias significativas en función de las variables: Años de experiencia docente $(p=0,247)$, tipos de espacio empleado $(p=0,470)$ y el nivel educativo $(p=0,521)$. Sin embargo, se han detectado diferencias significativas respecto al tipo de centro $(p=0,027)$, la especialidad $(p=0,000)$, la edad del tutor de prácticas $(p=0,000)$ y la formación pedagógica del/de la tutor/a $(p=0,016)$.

Una vez detectadas diferencias significativas entre las citadas variables, se procederá a profundizar en ello con el fin de determinar en qué pares de grupos se concretan las diferencias constatadas mediante una prueba de comparación múltiple. De acuerdo con Hernández-Veleros, Dolores y Amador (2011), los contrastes múltiples presentan un inconveniente asociado al nivel de significación, llamado de las comparaciones múltiples. Para definir el nivel de significación $((\alpha=0,05)$ en el estudio post-hoc de las diferencias de cada una de las variables, se recalculará el valor de a para cada contraste de pareja.

Para ello, empleando la aproximación de Bonferroni se calcula el nuevo valor al que llamaremos $\alpha^{\star}$. La fórmula para hacer este cálculo se basa en que $\alpha^{`}=\alpha / T$, donde $\mathrm{T}$ es el número de comparaciones o pruebas estadísticas posibles a realizar a posteriori (Hernández-Veleros et al., 2011; Wilkinson, 1999). Por tanto, en nuestro caso, a para la variable especialidad es 0,0083 ya que $0,0083=0,05$ / 6, siendo 6 el número de comparaciones específicas posibles. Análogamente, el valor de a para las variables tipo de centro, edad del/de la tutor/a y formación pedagógica del/de la tutor/a será: 0,009, 0.000 y 0,003, respectivamente.

Una vez establecido nuestro nivel de significación a correspondiente se procede a realizar la prueba $U$ de Mann-Whitney para cada par de grupos en los que se divide cada una de las diferentes variables que han resultado significativas, en la variable uso de Gamificación de acuerdo con la prueba de Kruskal Wallis. En las tablas de la 9 a la 12 se muestran los resultados obtenidos.

De acuerdo con dichos resultados, en relación a la especialidad, las diferencias estadísticamente significativas se presentan, con $p$ valores menores de 0,05 , en matemáticas respecto a Lengua extranjera Inglés, Lengua y Literatura y Geografía e Historia, respectivamente. Tales diferencias indican que el uso de la 
metodología en la especialidad de matemáticas es significativamente inferior respecto al resto de especialidades (Tabla 9).

Tabla 9. Diferencias por pares en la variable "uso de la Gamificación (GA) en función de la especialidad".

\begin{tabular}{lcc}
\hline Tipo de centro & U-Mann-Whitney & P-valor \\
\hline Matemáticas-Lengua & 401 & 0,0002 \\
Matemáticas-Inglés & 1024 & 0,0000 \\
Matemáticas-Historia & 1272 & 0,0002 \\
Lengua-Inglés & 334 & 0,7233 \\
Lengua-Historia & 397,5 & 0,6663 \\
Inglés-Historia & 1146 & 0,268 \\
\hline
\end{tabular}

NOTA: U es el estadístico U de Mann-Whitney; z es la aproximación a la normal; p la significación asintótica bilateral (nivel de significación de $0,05)$.

Con respecto al tipo de centro, las diferencias estadísticamente significativas se han detectado, con $p$-valor $=0,0002$, entre los colegios concertados y públicos (Tabla 10).

Tabla 10. Diferencias por pares en la variable "uso de la Gamificación (GA) en función del tipo de centro".

\begin{tabular}{lcc}
\hline Tipo de centro & U-Mann-Whitney & P-valor \\
\hline Privado-Concertado & 854 & 0,5119 \\
Privado-Público & 720 & 0,197 \\
Concertado-Público & 1272 & 0,0002 \\
\hline
\end{tabular}

NOTA: U es el estadístico U de Mann-Whitney; z es la aproximación a la normal; p la significación asintótica bilateral (nivel de significación de 0,05).

Las respuestas proporcionadas sobre el empleo de la Gamificación respecto a la edad del tutor indican que su uso es mayor significativamente en los profesores menores de 40 años (Tabla 11).

Tabla 11. Diferencias por pares en la variable "uso de la Gamificación (GA) en función de la edad del tutor".

\begin{tabular}{lcc}
\hline Tipo de centro & U-Mann-Whitney & P-valor \\
\hline Más de 40-Entre 25 y 40 años & 3526,5 & 0,0056 \\
\hline $\begin{array}{l}\text { NOTA: U es el estadístico U de Mann-Whitney; z es la aproximación a la normal; p la significación asintótica bilateral (nivel de significación de } \\
\text { 0,05). }\end{array}$
\end{tabular}

Por último, respecto a la formación pedagógica del tutor/a, hay que indicar que las diferencias más significativas estadísticamente se muestran entre disponer el CAP frente a tener el Máster del Profesorado y ninguna formación, respectivamente con $p$-valores menores de 0,05 . Poner en valor que disponer del CAP refleja un menor uso de la metodología en las aulas.

Tabla 12. Diferencias por pares en la variable "uso de la Gamificación (GA) en función de la formación pedagógica del tutor". 


\begin{tabular}{lcc}
\hline Tipo de centro & U-Mann-Whitney & P-valor \\
\hline CAP-Máster de Formación del profesorado & 2305 & 0,026 \\
\hline NOTA: U es el estadístico U de Mann-Whitney; z es la aproximación a la normal; p la significación asintótica bilateral (nivel de significación de \\
0,05).
\end{tabular}

\section{Discusión}

La investigación realizada en este trabajo se ha producido en un contexto de excepcionalidad derivado por la necesidad de los centros de compaginar la actividad educativa con las medidas sanitarias impuestas por las autoridades. Por ese motivo, a la hora de discutir los resultados de este trabajo, es importante señalar que estos pueden estar condicionados por la presencia de esta variable extraña.

En la tabla 1, se muestra que el uso de la gamificación es empleado en el $20 \%$ de las aulas observadas. Este dato supone un aumento de 8 puntos respecto a los datos publicados en Roa, Sánchez y Sánchez (2021) que señalaban que el uso de la gamificación durante el primer semestre del curso $2019-20$ eran del $12 \%$. Son varias las hipótesis que pueden explicar este aumento y que se deberían contrastar en futuros estudios.

Por un lado, son varios los autores: Cabero y Valencia (2020), Moreno-Correa (2020; Cotonieto et al. (2021), Díez y Gajardo (2020), que han señalado que durante la pandemia por COVID-19 se han producido numerosas transformaciones y adaptaciones ampliando el uso de recursos y medios digitales, por otro lado, la necesidad de respetar las medidas de distanciamiento social ha llevado a muchos centros a modificar la organización de espacios y tiempos lo que ha supuesto en muchos casos una reducción de ratios y una posibilidad de utilización de espacios diferentes al aula clase habitual.

Como se ha visto en la introducción, la gamificación necesita introducir dinámicas, mecanismos y componentes, en muchas ocasiones, estos elementos se apoyan en recursos digitales por lo que los cambios en los usos de recursos digitales podrían estar favoreciendo su implantación en secundaria. Adicionalmente el hecho de reducir las ratios, aunque no hay consenso en la comunidad científica sobre su eficacia general Sánchez (2018), puede ser una hipótesis explicativa para investigar en relación con el aumento de uso de aulas gamificadas detectado.

A pesar de este incremento general en la Tabla 2 podemos observar como la especialidad de matemáticas hace un uso significativamente menor que las especialidades de Lengua, Lengua extranjera y Geografía e Historia. Este hecho concuerda con lo encontrado en Roa et al. (2021) donde la especialidad de matemáticas era la que menos empleaba esta metodología. Sin embargo, es un hecho destacable, que mientras la frecuencia de uso aumenta en las especialidades de Lengua, Lengua extranjera y Geografía Historia respecto al curso 19-20 en la especialidad de matemáticas se ha producido un descenso notable. La literatura sobre los beneficios y usos de la gamificación en el ámbito STEM es muy extensa y presenta un gran número de publicaciones de impacto como se puede ver en el trabajo de Fuentes y González (2019) por lo que el uso significativamente menor en la asignatura de matemáticas unido al fracaso y falta de motivación asociado a esta materia es un resultado preocupante que debe dar lugar a futuros trabajos e investigaciones sobre cómo difundir esta metodología dentro de ese colectivo. 
Respecto a la tipología de centro se observa un uso significativamente menor en los centros de titularidad pública, este dato también fue señalado en Roa et al. (2021). En el lado positivo, se observa que el aumento general en el uso de la gamificación se ha producido en todos los tipos de centros. De forma global podemos señalar que, en este tema al igual que en otros como los señalados por Murillo, Belaví y Pinilla (2018), existen diferencias importantes entre centros públicos y concertados-privados que pueden afectar a la equidad del conjunto del sistema.

Adicionalmente a estos hallazgos, en nuestro estudio se han detectado dos variables a tener en cuenta y que influyen significativamente en el empleo de la gamificación, la edad del docente y la formación pedagógica recibida. En el análisis de la literatura sobre gamificación realizado por Trinidad, Ruiz y Calderón (2021) se analiza una muestra de 4706 publicaciones sobre gamificación en el intervalo 2011-2019 y se detectan varios clusters temáticos sobre el tema como el uso general de la gamificación en diferentes contextos, las relaciones entre los elementos de la gamificación y los efectos que producen en diferentes factores de la personalidad o la aplicación de la gamificación en educación. Es llamativo que en una revisión tan extensa no aparezcan estudios que hayan analizado la importancia de la formación docente o la edad a la hora de aplicar esta metodología.

En un estudio más limitado realizado por Valencia y Orellana (2019) sobre 101 trabajos de investigación, se señalan 5 tipos de barreras en la implementación de la gamificación en la educación superior: tecnológicas, docentes, estudiantes, diseño de la estrategia gamificada y pedagogía. Aunque en este caso se incluyen dentro de la categoría de barreras docentes 6 restricciones no aparecen entre ellas la edad y la formación. Este trabajo pone de manifiesto dos variables adicionales a la hora de poner en marcha esta metodología en la educación secundaria por lo que este hallazgo abre una línea de trabajo futura y aporta algunas claves para superar barreras en la implantación de la gamificación que pueden servir a la hora de difundir y poner en marcha acciones formativas en determinados colectivos docentes. Como hipótesis a valorar, el colectivo docente que más emplea esta metodología es menor de 40 años y ha sido el formado en el Máster de formación del profesorado por lo que su incorporación a la docencia y su etapa formativa se produjo a partir de la primera década del siglo XXI en plena expansión de la metodología.

Por último, la variable generacional que se aprecia en la tabla 7 invita a realizar estudios que relacionen la implantación de la gamificación con el cambio de paradigma señalado por Area y González (2015). El cambio de un sistema basado en el libro de texto y el conocimiento enciclopédico propio del siglo XX hacia un paradigma digital y competencial en línea con las demandas de la sociedad del Siglo XXI puede ser un aspecto explicativo de las diferencias encontradas según el grupo de edad.

\section{Conclusiones}

El sistema educativo se ha visto sacudido por una situación excepcional que ha provocado nuevos retos y desafíos y que ha obligado a los centros educativos a afrontar cambios importantes como la necesidad de llevar a cabo una enseñanza virtual y semipresencial o la obligación de trabajar bajo unas medidas sanitarias que dificultan algunos procesos como la colaboración entre estudiantes o el uso de materiales manipulativos. En este contexto en este trabajo se ha estudiado la implantación de la gamificación como metodología activa en las aulas de secundaria, bachillerato y FP en el sistema educativo con currículo español. 
Como conclusiones principales, podemos destacar que el uso de la gamificación ha aumentado respecto a la situación prepandemia y que se sitúa en el entorno del $20 \%$ de las aulas observadas. Sin embargo, su uso es muy diferente en función del tipo de centro, la materia o la formación y la edad del docente. En general se aprecia un mayor uso en los centros privados y concertados, en las asignaturas de Lengua, Lengua extranjera y Geografía e Historia y entre los docentes con formación profesional de Máster y con una edad inferior a los 40 años. En el lado opuesto, el uso de la gamificación en los centros públicos, en la materia de matemáticas y en aulas tutorizadas por profesores veteranos y formados mediante el CAP se detectan niveles de implantación significativamente menores.

Los resultados de este trabajo abren varias líneas interesantes de trabajo. Por un lado, abre la investigación hacia el papel que juega la experiencia, la edad y la formación de los docentes a la hora de implantar esta metodología. Por otro, se confirman ciertas tendencias encontradas antes de la Pandemia por COVID-19 como el mayor uso en los centros privados y concertados respecto a la pública o el bajo impacto de la metodología en la asignatura de matemáticas, que llevan a plantear la necesidad de realizar nuevos estudios longitudinales para medir la evolución de su implantación y el posible efecto distorsionador provocado por la situación de excepcionalidad vivida durante el curso 2020/21.

Para concluir, nos gustaría señalar que los beneficios y la evolución que está teniendo esta metodología son muy prometedores tal y como reflejan los múltiples estudios publicados. Sin embargo, y a tenor de lo encontrado en este trabajo, se empiezan a detectar resultados que ponen de manifiesto un posible problema de equidad en el sistema educativo con currículo español y que conlleva la necesidad de realizar nuevas investigaciones y mayores esfuerzos de divulgación y formación entre los colectivos que menos han avanzado en su uso.

\section{Referencias}

Alejaldre, L y García, AL (2015) Gamificar: el uso de los elementos del juego en la enseñanza de español. En Celma, MP, Gómez, MJ y Morán, $C$ (Eds.) Actas del $L$ congreso internacional de la AEPE (pp. 73-83).

Amezcua, T y Amezcua, P (2018). La gamificación como estrategia de motivación en el aula. En Torres, A y Romero, LM (Eds), Gamificación en Iberoamérica. Experiencias desde la Comunicación y la Educación (pp. 137-146).

Area, M y González, C (2015). De la enseñanza con libros de texto al aprendizaje en espacios online gamificados. Educatio Siglo XXI, Murcia, 33(3), 15-38.

Berger, VW, \& Zhou, Y (2014). Kolmogorov-Smirnov Test: Overview. In Wiley StatsRef: Statistics Reference Online (Eds N Balakrishnan, T Colton, B Everitt, W Piegorsch, F Ruggeri, \& JL Teugels). https://doi.org/10.1002/9781118445112.stat06558

Bruder, P (2015). Game on: Gamification in the Classroom. Education Digest, 80(7), 56-60.

Cabero Almenara, J (2015) Reflexiones educativas sobre las tecnologías de la información y la comunicación (TIC). Revista Tecnología, Ciencia y Educación, 1, 19-27.
Cabero, J y Valencia, R (2020). Y el COVID-19 transformó al sistema educativo: reflexiones y experiencias por aprender. International Journal of Educational Research and Innovation (UERI), 15, 218-228.

Cotonieto-Martínez, E, Martínez-García, R y Rodrigo, T (2021). Reflexiones sobre la educación en tiempos de COVID-19: retos y perspectivas. Revista saberes educativos, 6, 116-127.

Deterding, S, Dixon, D, Khaled, R, \& Nacke, L (2011). From game design elements to gamefulness: Defining "gamification". En Memorias del 15th International Academic MindTrek Conference: Envisioning Future Media Environments (pp. 9-15). https://doi.org/10.1145/2181037.2181040

Díaz, PP (2017). Gamificando con Kahoot en evaluación formativa. Revista Infancia, Educación y Aprendizaje, 3(2), 112-117.

Díez, EJ y Gajardo, K (2020). Educar y evaluar en tiempos de coronavirus: la situación en España. Multidisciplinary Journal of Educational Research, 10(2), 103-127.

Fuentes, M y González, J (2019). Qué gana STEM con la gamificación. Revista Academia y virtualidad, 12(2), 79-94. 
Gallego Aguilar, AF y Ágredo Ramos, AF (2016). "Implementando una metodología de gamificación para motivar la lectura y escritura en jóvenes universitarios." Kepes, 14, 61.

García, CM (2016). La senda del maestro: experiencias de gamificación en el aula universitaria. Comunicación del XII "Congreso Español de Sociología. Grandes transformaciones sociales, nuevos desafíos para la sociología".

González, Olivares, García y Figueroa (2017) Propuesta de Gamificación en el aula: Uso de una plataforma para motivar a los estudiantes del Programa Académico de Informática de la Universidad Autónoma de Nayarit. Revista Educateconciencia. 13, $14,70-79$

González Reyes, JA, Olivales Granados, SA, García Sánchez, E y Figeroa Melchor, IG (2017). Propuesta de gamificación en el aula: Uso de una plataforma para motivar a los estudiantes del Programa Académico de Informática de la Universidad Autónoma de Nayarit. Educateconciencia, 13(14), 70-79.

Kapp, KM (2012). The Gamification of Learning and Instruction: Game-Based Methods and Strategies for Training and Education. New York: Pfeiffer.

Ley Orgánica 8/2013

Marczewski, A. (2013). Gamification: A Simple Introduction and a Bit More, (self-published on Amazon Digital Services, 2013). Kindle edition, Loc, 1405

Marczewski, A (2015). Even Ninja Monkeys Like to Play: Gamification, Game Thinking and Motivational Design. California: CreateSpace Independent Publishing Platform.

McKnight, PE, \& Najab, J (2010). Mann-Whitney U Test. In The Corsini Encyclopedia of Psychology (eds I.B. Weiner and W.E. Craighead).

Moreno-Correa, S. M. (2020). La innovación educativa en los tiempos del Coronavirus. Salutem Scientia Apiritus, 6(1), 14-26.

Murillo, FJ, Belavi, G y Pinilla, L (2018) Segregación escolar público-privada en España. Papers: revista de sociología, 103(3), 307-337.

Olivares, S y González, J (2016). La generación Z y los retos del docente. En Velasco, M. Páez, (eds.). Los retos de la docencia ante las nuevas características de los estudiantes universitarios.

Ordás, A (2018). Gamificación en bibliotecas: El juego como inspiración. Barcelona: Ed. UOC.

Ortiz Carpintero, T (2017). Gamificación: La vuelta al mundo en 80 días. Revista Infancia, Educación y Aprendizaje, 3(2), 397-403.

Pérez López, IJ, Rivera García, E y Trigueros Cervantes, C (2017). "La profecía de los elegidos": un ejemplo de gamificación aplicado a la docencia universitaria / "The Prophecy of the Chosen Ones": An Example of Gamification Applied to University Teaching. Revista Internacional de Medicina y Ciencias de la Actividad Física y el Deporte, 17(66), 243-260

Pérez de Villaamil, T (2018). Gamificación en el aula: ventajas y desventajas. https://www.smartmind.net/blog/gamificacion-en-elaula-ventajas-y-desventajas/
Quintanal Perez, F (2016). Gamificación y la Física-Química de Secundaria. Education in the Knowledge Society (EKS), 17(3), 1328. https://doi.org/10.14201/eks20161731328

Roa González, J, Sánchez Sánchez, A y Sánchez Sánchez, N (2021). Evaluación de la implantación de la Gamificación como metodología activa en la Educación Secundaria española. REIDOCREA, 10(12), 1-9. https://doi.org/10.30827/Digibug.66357

Sánchez, E (2018). El salario del profesorado y el tamaño de la clase: dos indicadores sugerentes para una reforma educativa de calidad. Revista Complutense de Educación. 29(1), 199-213.

Trinidad, M, Ruiz, M, \& Calderón, A (2021). A Bibliometric Analysis of Gamification Research. IEEE Access, 9, 46505-46544.

Valencia, L y Orellana, D (2019). Barreras en la implementación de la gamificación en educación superior: Revisión de Literatura. In Crescendo 10(4), 635-650.

Werbach, K, \& Hunter, D (2012). For the win: How game thinking can revolutionize your business. Philadelphia, PA. Wharton School Press.

Werbach, K, \& Hunter, D (2015). The gamification toolkit: Dynamics, mechanics, and components for the win. Philadelphia, PA. Wharton School Press.

Wiklund, E, \& Wakerius, V (2016). The Gamification Process: A framework on gamification. Student thesis. Jönköping University.

Kruskal, WH, \& Wallis, WA (1952). Use of ranks in one-criterion variance analysis. Journal of the American statistical Association, 47(260), 583-621.

Zichermann, G, \& Cunningham, C (2011). Gamification by design: Implementing game mechanics in web and mobile apps. Canada: o'Reilly Media. 